Gynäk. Rdsch. 1973;13(Suppl. 1):63

\title{
Hormonausscheidung unter Minipille
}

\begin{tabular}{|l|l|}
\hline L. & Varga \\
\hline E. & Tamme \\
\hline
\end{tabular}

Abteilung für Gynäkologie und Geburtshilfe (Chefarzt: Prof. R. Wenner), Kantonsspital Liestal

\section{Zusammenfassung}

Bei 9 Probandinnen wird die Beeinflussung der Gonadotropin-Ovarialfunktion unter Dauergabe von 0,35 mg Norethisteron (Cilag) bzw. 0,5 mg Lynestrenol (Orga-non) als Minipille untersucht. Als Kriterien werden die Basaltemperaturmessung

die LH-

Gesamtöstrogen- und Pregnandiolausscheidung verwendet. Zu Beginn der Einnahme stellt sich gewöhnlich eine Ovulationshemmung ein

die jedoch in der Regel mit Fortschreiten der Einnahme zurückgeht. Eine Lutealinsuffizienz scheint auch weiterhin zu bestehen.

1 Publikation in Vorbereitung. 